\begin{tabular}{|l|l|}
\hline $\begin{array}{l}\text { Instituto de } \\
\text { Geriatria e Gerontologia }\end{array}$ & $\begin{array}{l}\text { Pan American Journal of Aging Research } \\
\text { PAJAR, Porto Alegre, v. 8, p. 1-8, jan.-dez. } 2020 \\
\text { ISSN-L: 2357-9641 }\end{array}$ \\
\hline di) ${ }_{\text {http://dx.doi.org/10.15448/2357-9641.2020.1.35631 }}$ &
\end{tabular}

\title{
Aplicação do MINICHAL em um grupo de idosos hipertensos vinculados ao setor de saúde suplementar
}

\author{
Application of MINICHAL in a group of elderly hypertensive perteding to the \\ supplemental health sector \\ Aplicación de MINICHAL en un grupo de ancianos hipertensos vinculados al sector de \\ salud suplementario
}

\section{Henrique Souza Barros de Oliveira ${ }^{1}$ \\ orcid.org/0000-0002-9067-8581 \\ heeenry.barros@icloud.com}

\section{Leticia Silva de Jesus ${ }^{1}$}

orcid.org/0000-0002-2060-5387

leticiasjs@hotmail.com

Diego Reses de Gino

orcid.org/0000-0002-1842-7921 resesdiego@gmail.com

\section{Maria Elisa Gonzalez \\ Manso ${ }^{1}$}

orcid.org/0000-0001-5446-233X

mansomeg@hotmail.com

Recebido em: 11 set. 2019 Aprovado em: 27 jan. 2020. Publicado em: 03 agos.. 2020

\section{(c) (1)}

Artigo está licenciado sob forma de uma licença Creative Commons Atribuição 4.0 Internacional.

\section{Resumo}

Objetivo: aplicar o instrumento MINICHAL para mensurar o perfil de qualidade de vida de um grupo de idosos hipertensos assistidos pelo setor de saúde suplementar.

Métodos: foram investigados 47 idosos hipertensos vinculados a uma operadora de planos de saúde, no municipio de São Paulo, SP. Brasil. Os dados sociodemográficos, clínicos, de utilização de serviços de saúde e de Qualidade de Vida relacionada à Saúde (QVRS) foram obtidos por meio de instrumentos criados pelos próprios pesquisados e da aplicação da versão brasileira do MINICHAL. Após expressas as variáveis descritivas, os testes de t-Student e Mann-Whitney foram realizados para comparar QVRS as demais variáveis.

Resultados: o MINICHAL indicou baixo comprometimento da QVRS em todas as dimensões do instrumento utilizado e a influência negativa entre a prática de atividade física e a dimensão Manifestações Somáticas $(\mathrm{p}=0,017)$.

Conclusão: o MINICHAL mostrou-se eficaz na mensuração da QVRS de idosos portadores de Hipertensão Arterial Sistêmica vinculados ao setor de saúde suplementar e evidenciou um menor comprometimento na QVRS entre os idosos praticantes de atividade física.

Palavras-chave: idoso, hipertensão arterial sistêmica, qualidade de vida, planos de pré-pagamento em saúde.

\section{Abstract}

Objective: to apply the MINICHAL instrument to measure the quality of life profile of a group of hypertensive elderly people assisted by the supplementary health sector.

Methods: we investigated 47 hypertensive elderly people linked to a health plan operator in the city of São Paulo, SP, Brazil. Sociodemographic, clinical, health service utilization and Health Related Quality of Life (HRQoL) data were obtained through instruments created by the respondents themselves and the application of the Brazilian version of MINICHAL. After the descriptive variables were expressed, the Student $t$ and Mann-Whitney tests were performed to compare HRQoL to the other variables.

Results: MINICHAL indicated low HRQL impairment in all dimensions of the instrument used and the negative influence between physical activity practice and the "Somatic Manifestations" dimension ( $\mathrm{p}=0.017$ ).

Conclusion: MINICHAL was shown to be effective in the measurement of HRQOL of elderly people with hypertension linked to the supplementary health sector and evidenced a lower impairment in HRQOL among the elderly practicing physical activity.

Keywords: elderly, hypertension, quality of life, prepaid health plans. 
Resumen

Objetivo: aplicar el instrumento MINICHAL para medir el perfil de calidad de vida de un grupo de ancianos hipertensos atendidos por el sector de salud suplementario. Métodos: investigamos a 47 ancianos hipertensos vinculados a una compañía de seguros de salud en la ciudad de São Paulo, SP, Brasil. Los datos sociodemográficos, clínicos, de servicios de salud y de Calidad de Vida Relacionada con la Salud (CVRS) se obtuvieron a través de instrumentos creados por los propios encuestados y aplicando la versión brasileña de MINICHAL. Después de expresar las variables descriptivas, se realizaron las pruebas t-Student y Mann-Whitney para comparar la CVRS con las otras variables.

Resultados: el MINICHAL indicó un bajo deterioro de la CVRS en todas las dimensiones del instrumento utilizado y la influencia negativa entre la práctica de actividad fisica y la dimensión "Manifestaciones Somáticas" ( $p=0.017$ ). Conclusiones: el MINICHAL fue eficaz para medir la CVRS de ancianos con hipertensión relacionada con el sector de la salud suplementario y evidenció un menor deterioro en la CVRS entre los ancianos que practican actividad física.

Palabras clave: anciano, hipertensos, calidad de vida, planos de salud de prepago.

\section{Introdução}

A Hipertensão Arterial Sistêmica (HAS) é uma enfermidade de manifestação silenciosa, multifatorial, com caráter crônico degenerativo e de alta prevalência, principalmente entre os idosos. ${ }^{1}$ Além disso, a HAS é um dos principais fatores de risco associado ao desenvolvimento de doenças cardiocirculatórias, tais como cardiomiopatia dilatada, cardiomiopatia isquêmica, acidente vascular encefálico e doença renal crônica. ${ }^{2}$

Estudos vêm mostrando o impacto direto da presença de Doenças Crônicas Não Transmissiveis (DCNT) sobre a Qualidade de Vida Relacionada à Saúde (QVRS) através da utilização de instrumentos amplos de mensuração. ${ }^{3-6}$ No entanto, poucos utilizam instrumentos especificos em grupos geriátricos, sendo que entre 50 a $70 \%$ dos idosos são portadores de HAS.? Essa escassez se acentua para o contexto do setor suplementar de saúde, sendo que os idosos correspondem a maior parcela de pessoas presentes nas carteiras de planos de saúde no Brasil. ${ }^{8}$

Investigar a QVRS em grupos de diferentes contextos possibilita futuras comparações e o planejamento de estratégias de intervenção, tanto por órgãos governamentais quanto pelos próprios serviços de saúde e operadoras de convênios médicos.
Diante do exposto, este estudo teve como propósito mensurar o perfil da qualidade de vida e variáveis associadas ao construto em um grupo de idosos hipertensos vinculados a uma operadora de plano de saúde, localizada na cidade de São Paulo, SP, Brasil.

\section{Métodos}

\section{Delineamento da pesquisa}

Estudo descritivo, de corte transversal analítico realizado com um grupo de idosos portadores de HAS, todos participantes de um programa de gerenciamento de doenças crônicas degenerativas oferecido por uma operadora de planos de saúde, modalidade medicina de grupo, localizada na cidade de São Paulo, SP, Brasil.

\section{Sujeitos da pesquisa e critérios de exclusão}

Neste estudo, foram incluidos 47 idosos, independentemente do sexo, bastando que tivessem idade igual ou superior a 60 anos completos e diagnóstico médico prévio de HAS. Em razão das particularidades dos questionários utilizados neste estudo, autopreenchiveis, foram excluidos idosos com déficit cognitivo, inconscientes, institucionalizados e sem condições clinicas para responder por si próprios aos questionários.

\section{Processo de amostragem}

A amostragem foi realizada de forma aleatória simples dentro do total de 54 idosos hipertensos participantes do programa de gerenciamento, com grau de confiança de 95\% para o cálculo amostral.

\section{Instrumentos}

Para esta pesquisa, foram utilizados os instrumentos (1) questionário estruturado para coleta de variáveis sociodemográfica e do estado de saúde; e (2) a versão brasileira do instrumento MINICHAL para avaliar a QVRS dos idosos portadores de HAS. ${ }^{9}$

A fim de caracterizar o perfil sociodemográfico, clínico e de utilização dos serviços de saúde, os pesquisadores elaboraram um questionário estruturado com o intuito de coletar informações referentes a: sexo; idade; condição civil e ocupacional; se mora sozinho ou não; se possui 
cuidador; presença de morbidades crônicas e fatores de risco em tratamento até o momento da entrevista; se possui médico específico de referência; o periodo da última consulta médica; presença de internações hospitalares prévias; 0 hábito de fumar; sedentarismo; o consumo de álcool pelo Teste de Identificação de Distúrbio de Uso do Álcool (AUDIT) ${ }^{10 ;}$ e o Índice de Massa Corpórea (IMC), de acordo com padrões de corte preconizados para a idade..$^{11}$

Para a avaliação da QVRS foi utilizado o MINICHAL, na versão traduzida e validada no Brasil, por ser um questionário específico para avaliação da qualidade de vida a individuos hipertensos, de fácil e rápida aplicação. ${ }^{9}$ Trata-se de um instrumento autoadministrado, composto por 17 questões divididas nas dimensões Estado Mental (1 a 9; pontuação máxima de 27 pontos), Manifestações Somáticas (10 a 16; pontuação máxima de 21 pontos) e uma questão geral de qualidade de vida que não se inclui em nenhuma das dimensões anteriores. ${ }^{9}$

Cabe salientar que para cômputo dos escores, foi utilizado o preconizado pela versão brasileira do MINICHAL onde a questão 10 é somada a dimensão Manifestações Somáticas, diferentemente da versão original do instrumento. A questão sobre a percepção geral de saúde não foi considerada no cômputo do score total, a exemplo do estudo de validação.9.12

Os itens foram respondidos em referência aos últimos sete dias antecedentes à aplicação do instrumento. A escala de pontuação é do tipo Likert com quatro possiveis respostas: $0=$ não, absolutamente; 1 = sim, um pouco; 2 = sim, bastante; e $3=\mathrm{sim}$, muito. Os pontos variam de $\mathrm{o}$ (melhor nivel de saúde) a 27 (pior nivel de saúde) para as dimensões Estado Mental, já para a dimensão Manifestações Somáticas, variam de o (melhor nivel de saúde) a 21 (pior nivel de saúde). Ao final, cada dimensão do instrumento foi representada em scores percentuais de zero a 100. ${ }^{9}$

\section{Coleta de dados}

A coleta dos dados ocorreu através de visitas domiciliares, agendadas previamente por telefone. Durante o agendamento, todos os idosos receberam explicações completas a respeito da pesquisa e ficaram cientes da participação voluntária, que sua não aceitação não interferiria na atenção prestada pelo plano de saúde ou desligamento do programa, e que os seus nomes e dados seriam preservados em sigilo. No momento da entrevista, o Termo de Consentimento Livre e Esclarecido foi assinado.

Os dados coletados foram, inicialmente, armazenados em um banco de dados próprio da pesquisa e submetidos à revisão e à codificação. Em casos de inconsistência, procedia-se correção das informações colhidas por meio da consulta à entrevista original.

Quanto às respostas obtidas através do instrumento autoadministrado MINICHAL, essas foram consolidadas em banco com as suas respectivas sintaxes.

\section{Análise dos dados}

Todos os dados coletados foram transferidos para o software Excel for Windows 2003 e transportados para o software Statistical Package for Social Sciences (SPSS ${ }$ - Chicago, EUA) for Windows, version 23.0, para análise descritiva e analítica.

Para análise estatística, os dados contínuos foram expressos na forma de medidas de tendência central e de dispersão, enquanto os dados categóricos foram expressos por distribuição de frequência absoluta e relativa. Para análise comparativa de associação, utilizou-se o teste $t$-Student para análise bivariada e o teste não paramétrico de Mann-Whitney para verificar associação em grupos que havia poucos participantes (<10 idosos). Considerou-se o nivel de significância estatística entre os grupos quando $p$-valor foi menor do que 0,05 ( $p$-valor $<0,05)$ para todos os testes realizados.

\section{Questões éticas}

O projeto de pesquisa foi aprovado pelo Comitê de Ética em Pesquisa (CEP) da Pontificia Universidade Católica de São Paulo (PUC-SP), sob n. do parecer 2.284.626, atendendo às normas nacionais e internacionais de ética em pesquisa envolvendo seres humanos (Resolução 466/2012). 


\section{Resultados}

\section{Caracteristicas sociodemográficas}

Participaram deste estudo 47 idosos hipertensos, residentes na cidade de São Paulo, SP, Brasil e vinculados a um programa de gerenciamento de doenças crônicas oferecido por uma operadora de planos de saúde. A amostra, em sua maioria, foi constituida por idosas do sexo feminino $(46,9 \%$; 22), cuja média de idade foi igual a $72 \pm 6$ anos. Quanto à distribuição por estado civil e situação ocupacional, 72,3\% eram casados ou viviam em situação de união estável e 79,5\% referiram ser aposentados.

Ressalta-se que $18,2 \%$ dos idosos desse grupo moram sozinhos e apenas um único idoso possui cuidador.

\section{Caracteristicas clinicas e potenciais fatores de risco}

Inquiridos sobre as principais comorbidades crônicas e fatores de risco associados à HAS, a presença de diabetes mellitus foi a mais prevalente (31,9\%; $n=15)$, seguida de dislipidemia (53,2\%; $n=25)$, tireoidopatia (23,4\%; $n=11)$, sobrepeso $(36,2 \% ; n=17)$ e obesidade ( $34 \% ; n=16)$. Encontrou-se, ainda, que $59,6 \%$ do grupo estudado possui mais de 3 morbidades associadas atualmente em tratamento.

Quanto ao hábito de fumar, 95,7\% dos idosos desse grupo não eram tabagistas, porém, 57,4\% relataram ser sedentários. Quanto à realização do AUDIT, 23,4\% apresentaram provável risco para dependência.

\section{Utilização dos serviços de saúde}

Neste grupo, identificou-se que, a totalidade da amostra estudada fazia acompanhamento médico para tratar as suas morbidades, sendo que nos últimos seis meses, $48,9 \%$ realizaram ao menos uma consulta médica e 51,1\% realizaram consulta há, no máximo, dozes meses. Quanto a internações hospitalares prévias, 57,4\% descreveram que em algum momento da vida já foram internados, sendo que 19,1\% foram internados uma vez no último ano por causas diversas, não relacionadas à HAS.

\section{QVRS e associações comparativas}

A média da QVRS mensurada pelo score total do MINICHAL em uma escala de 0 a 100 , foi de 40,1 $\pm 18,2$. As médias por dimensões foram bastante próximas, sendo na dimensão Estado Mental de 40,3 \pm 18 ,6 e na dimensão Manifestações Somáticas de 39,8 $\pm 18,6$. Quanto à última questão do instrumento, que avalia o impacto da HAS e seu tratamento sobre à QVRS, notou-se que $86,6 \%$ $(n=39)$ dos idosos consideraram que a hipertensão afeta de maneira significativa a sua QVRS.

Em relação às análises comparativas entre as dimensões do MINICHAL e as caracteristicas sociodemográficas, clinicas, fatores de risco e a utilização dos serviços de saúde, não foram encontradas associações significativas $(p>0,05)$ entre os idosos estudados, exceto a associação de realização de atividade fisica com menores scores na dimensão Manifestações Somáticas $(p=0,017)$.

\section{Discussão}

Os dados sociodemográficos de idosos vinculados ao setor de saúde suplementar não divergiu com os encontrados neste estudo, cuja predominância era de mulheres idosas, com idade igual ou superior a 70 anos, casadas, aposentadas e que residiam nos principais municipios da região Sudeste. ${ }^{13-17}$

Em virtude do processo fisiológico de envelhecimento, os idosos possuem maior predisposição ao aumento de doenças cardiovasculares e de suas complicações. As principais modificações que corroboram para esse fato são: (i) aterosclerose; (ii) aumento do acúmulo de gordura; (iii) diminuição da secreção de hormônios contrarreguladores; (iv) enrijecimento e envelhecimento dos vasos sanguíneos; (v) hipertrofia dos miócitos e da massa ventricular; (vi) progressivo aumento da resistência vascular periférica; e (vii) redução das respostas reflexas, dentre outros. ${ }^{11.18}$

Essas modificações, quando associadas a fatores de risco tais como os relacionados à idade, sexo, hábitos alimentares e sedentarismo, fazem com que ocorra um incremento não só da prevalência, mas da mortalidade relacionada às doenças cardiovasculares em idosos. ${ }^{17}$ Além da 
HAS, esse grupo de idosos pesquisado apresenta frequência de dislipidemia, Diabetes Mellitus e sedentarismo superior a outros estudos conduzidos com grupos populacionais semelhantes ${ }^{13,14,17}$, o que torna o grupo altamente vulnerável a eventos cardiovasculares potencialmente fatais.

A avaliação da QVRS através do instrumento MINICHAL é de extrema relevância para pesquisadores, profissionais clínicos e gestores, pois, se trata de um instrumento que possibilita mensurar o impacto psicossocial que a HAS e o seu tratamento podem acarretar, tanto positiva quanto negativamente, em grupos de distintos contextos, inclusive, indicando fatores que podem influenciar a sensação de bem-estar do indivíduo. Neste grupo, observou-se que as médias do score total, das dimensões Estado Mental e Manifestações Somáticas foram semelhantes aos outros estudos conduzidos com pacientes adultos e idosos hipertensos. ${ }^{1,2,9,12,19,20,21}$ Os resultados mensurados pelo MINICHAL indicam comprometimento da QVRS em todas as dimensões do instrumento, mostrando que a HAS e o seu tratamento geram impacto significativo na qualidade de vida desse grupo.

Estudos mostram que indivíduos hipertensos possuem uma diminuição significativa da QVRS quando comparados com normotensos e a principal explicação é que a presença de condições crônicas atua de maneira considerável nos aspectos psicossociais, limitando a sua interação com o meio ambiente e a sociedade. ${ }^{2}$

A literatura aponta que o aumento da idade atua como fator relevante para a redução dos scores da qualidade de vida, devido às modificações que se acentuam com o processo do envelhecimento. ${ }^{22,23}$ Deve-se considerar, ainda, que o grupo investigado, além da elevada média de idade, possui comorbidades em tratamento simultâneo, o que pode afetar negativamente a avaliação de sua qualidade de vida.

Como evidenciado nos resultados, o número elevado de idosos portadores de comorbidades crônicas utilizando os serviços de saúde repetidas vezes pode revelar, dentre outros fatores, o inadequado seguimento da terapia farmaco- lógica, importante fator de influência sobre a QVRS. ${ }^{24}$ Estudos apontam que três quartos dos indivíduos com HAS não atingem um controle da sua pressão arterial utilizando tratamento anti-hipertensivo. ${ }^{25}$ Nos idosos, a polifarmácia, a interação medicamentosa e os efeitos adversos do tratamento podem estar associadas a menor aderência e ao abandono do tratamento medicamentoso, além de corroborar para o aumento da procura dos serviços de saúde, descompensação das doenças de base, internações hospitalares recorrentes, aumento de sua permanência e a ocorrência de eventos iatrogênicos, inferindo de maneira significativa em suas QVRS. ${ }^{24}$

Além de medidas farmacológicas para o manejo do idoso hipertenso, devem ser instituidas também medidas não farmacológicas, que incluem a prática de atividade física, a suspensão do tabagismo e etilismo, a manutenção de dieta hipossódica e o controle do peso. ${ }^{1}$ No grupo pesquisado, observa-se um número significativo de idosos que se apresenta com IMC acima do recomendado e sedentarismo.

Analisando-se apenas o IMC, de acordo com os padrões de corte para idade, notou-se que uma minoria dos idosos estavam com o peso dentro dos padrões normais (29,8\%). Dado relevante, pois o aumento expressivo na prevalência da HAS ocorre em conjunto com o aumento do sobrepeso e da obesidade, sendo que aproximadamente $75 \%$ dos casos de hipertensão em homens e $65 \%$ em mulheres são atribuidos à obesidade. ${ }^{25}$ Além disso, o excesso de peso eleva a incidência de morbidades como Diabetes Mellitus do tipo 2 (DM), dislipidemia, aumento de marcadores inflamatórios, tromboembolismo e apneia do sono implicando em maior incidência de Doença Cardíaca Isquêmica (DCI), Arterial Coronariana (DAC), Acidente Vascular Encefálico (AVE), risco de morte súbita e da mortalidade cardiovascular e total. ${ }^{26}$ Além do que, a população geriátrica deve receber atenção redobrada, em virtude do aumento gradual de tecido adiposo visceral que ocorre com a perda de parênquima em diversos órgãos, decorrente do processo normal do envelhecer. ${ }^{11}$ Novamente se ressalta a presença 
e a interligação de fatores de risco que tornam esse grupo pesquisado propenso a apresentar eventos cardiovasculares graves.

Evidências cientificas apontam que a QVRS de idosos hipertensos está associada a fatores socioeconômicos e demográficos, como idade, gênero, estado conjugal, nível educacional e emprego. ${ }^{27.28 .29}$ A compreensão entre esses fatores é útil para subsidiar programas e medidas de ações em saúde que minimizem o impacto da HAS e o seu tratamento na vida de idosos. No entanto, em divergência com a literatura, o grupo estudado não apresentou associações estatisticamente significativas em nenhuma das variáveis sociodemográficas citadas acima. Contudo, o sedentarismo mostrou-se como única variável a influenciar negativamente a QVRS dessas pessoas idosas.

Sabe-se que, a realização de atividade física regular promove a diminuição dos niveis pressóricos de maneira progressiva, devido à redução da atividade simpática periférica e do tônus simpático cardiaco, que, por conseguinte, leva à diminuição da frequência cardiaca e à queda do débito cardiaco. A prática de exercícios fisicos deve sempre ser indicada como medida de prevenção, de retardo de perdas funcionais, melhora de fatores de risco cardiovasculares e de possiveis desfechos negativos e indesejados. Esses beneficios independem de fatores sociodemográficos, porém, dependem da intensidade, da duração e do tipo de atividade empregada. ${ }^{19.30}$

\section{Limitações}

Algumas limitações desta pesquisa merecem ser assinaladas. Os resultados apresentados merecem interpretações cautelosas, impossibilitando a sua generalização e a comparação com outros grupos, devido às particularidades do grupo e ao tamanho da amostra estudada. Além disso, deve-se levar em consideração a presença de viés nos dados encontrados, em virtude da seleção amostral por conveniência. Outra limitação se refere às próprias características do instrumento MINICHAL, pois, trata-se de um instrumento de autorrelato, portanto sujeito a viés de resposta.

\section{Conclusões}

O instrumento MINICHAL mostrou-se eficaz na mensuração da QVRS deste grupo de idosos hipertensos e indicou que, para os participantes deste estudo, a HAS e o seu tratamento geram impacto significativo na qualidade de vida.

Trata-se de um grupo caracterizado pela predominância do sexo feminino, idade elevada, presença de múltiplas comorbidades e de outros fatores de risco tais como sedentarismo, excesso de peso e obesidade, associados à HAS, o que leva a pensar que se trata de um grupo com alto risco para eventos cardiovasculares graves. Das variáveis que influenciam sua QVRS, apenas o sedentarismo se mostrou como sendo importante e que interfere negativamente nessa, evidenciando, assim, um menor comprometimento na QVRS entre os idosos praticantes de atividade fisica.

\section{Conflitos de interesse}

Os autores declaram não haver conflitos de interesse.

\section{Referências}

1. Maciel APF, Pimenta AB, Caldeira AP. Qualidade de vida e adesão medicamentosa para pessoas hipertensas. Act Paul Enferm. 2016;29(5):542-48. https://doi. org/10.1590/1982-0194201600075.

2. Carvalho MAN, Silva IBS, Ramos SBP, Coelho LF, Gonçalves ID, Figueiredo Neto JA de. Qualidade de vida de pacientes hipertensos e comparação entre dois instrumentos de medida de QVRS. Arq Bras Cardiol. 2012;98(5):442-51. https://doi.org/10.1590/S0066782X2012005000032.

3. Pilger C, Santos ROPS, Lentsck MH, Marques S, Kusumota L. Bem estar espiritual e qualidade de vida de idosos em tratamento hemodialítico. Rev Bras Enferm. 2017:70(4):721-29.

4. Pereira RMP, Batista MA, Meira AS, Oliveira MP, Kusumota L. Qualidade de vida de idosos com doença renal crônica em tratamento conservador. Rev Bras Enferm. 2017:70(4):887-95.

5. Amaral TLM, Amaral CA, Lima NS, Herculano PV, Prado PR, Monteiro GTR. Multimorbidade, depressão e qualidade de vida em idosos atendidos pela estratégia de saúde da família em Senador Guiomard, Acre, Brasil. Ciênc. Saúde Coletiva. 2018;23(9):3077-84. https://doi. org/10.1590/1413-81232018239.22532016.

6. Lima LR, Funghetto SS, Valpe CRG, et al. Qualidade de vida e o tempo do diagnóstico do diabetes mellitus em idosos. Rev Bras Geriatr Gerontol. 2018; 21(2):180-90. 
7. Andrade JMO, Rios LR, Teixeira LS, Vieira FS, Mendes DC, Vieira MA. Influência de fatores socioeconômicos na qualidade de vida de idosos hipertensos. Cienc Saúde Colet. 2014;19(8):3497-3504. https://doi. org/10.1590/1413-81232014198.19952013.

8. Manso MEG, Maresti LTP, Osti AV. Program for prevention of falls among elders: experiences in a Brazilian health plan. MOJ Gerontol Ger. 2018;3(2):217-218. https:// doi.org/10.15406/mojgg.2018.03.00114.

9. Schulz RB, Rossignoli P, Correr CJ, Fernández-Llimós F. Toni PM de. Validação do mini-questionário de Qualidade de vida em hipertensão arterial (MINICHAL) para o português (Brasil). Arq Bras Cardiol. 2010;90(2):127-131. https://doi.org/10.1590/S0066-782X2008000200010.

10. Portugal. Serviço de Intervenção nos Comportamentos Aditivos e nas Dependências (SICAD). Serviço de Intervenção nos Comportamentos Aditivos e nas Dependências. Serviço Nacional de Saúde [Internet]. Lisboa; 2015. [capturado 2018 dez 30]. Disponivel em: http://www.sicad.pt/PT/Intervencao/RedeReferenciacao/Site Pages/Instrumentos.aspx.

11. Pascali C, Peliello LC. Avaliação Nutricional. In: Manso MEG, organizador. Geriatria, Manual da Liga de Estudos do Processo de Envelhecimento. $1^{\text {a }}$ ed. São Paulo, SP: Martinari; 2015. p. 91-104

12. Badia X, Roca-Cusachs A, Dalfó A, Gascón G, Abellán J, Lahoz R, Varela C, Velasco O; MINICHAL Group. Validation of the short from of the Spanish hypertension quality of life questionnaire (MINICHAL). Clin Ther. 2002; 24(12):213754. https://doi.org/10.1016/S0149-2918(02)80103-5.

13. Manso MEG, Sousa JRP, Oliveira HSB. Analise do perfil neurogeriátrico de um grupo de idosos pertencentes a um plano de saúde do município de São Paulo, Brasil. Rev Kairós: Gerontol. 2018; 21(2):215-26.

14. Manso MEG, Osti AV, Borrozino NF, Maresti LTP. Avaliação multidimensional do idoso: resultados em um grupo de individuos vinculados a uma operadora de planos de saúde. Rev Kairós. 2018;21(1):191-211.

15. Oliveira HSB, Sousa JRP, Donis ACG, et al. Utilização dos critérios de Beers para avaliação das prescrições em idosos portadores de doenças crônicas vinculadas a um plano de saúde. RBCEH. 2017:14(3):242-251. https:// doi.org/10.5335/rbceh.v14i3.7376.

16. Manso MEG, Câmara R, Souza AS, Maciel TD, Farina DBL. Programa de gerenciamento de doenças crônicas em um plano de saúde, São Paulo, Brasil. Cienc Cuid Saude. 2016;15(2):321-27. https://doi.org/10.4025/ cienccuidsaude.v15i2.28683.

17. Manso MEG, Osti AV, Maresti LTP, Borrozino, NF. Análise do gerenciamento de doenças crônicas em uma operadora de planos de saúde na cidade de São Paulo. SODEBRAS. 2018:13(151):77-82.

18. Lisboa IAST, Perossi JT. O processo normal do envelhecer. In: Manso MEG, organizador. Geriatria, Manual da Liga de Estudos do Processo de Envelhecimento.

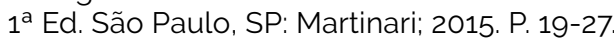

19. Gomes DBC, Bezerra EMA, Silva JCA, Costa TPS, Ribeiro MDA, Campelo GO. Avaliação da qualidade de vida em idosos hipertensos atendidos em clínica de fisioterapia. SANARE. 2015;14(01):33-7.

20. Soutello ALS, Rodrigues RCM, Jannuzzi FF, São-João TM, Martini, GG, Nadruz JrW, Gallani MBJ. Qualidade de vida na hipertensão arterial: validade de grupos conhecidos do MINICHAL. Arq Bras Cardiol. 2015; 104(4):299-307.

21. Côrtes DCS, Arantes AA, Mendonça APP, Silva JS. Qualidade de vida e hipertensão arterial. Int J Cardiovasc Sci. 2016;29(6):512-16.

22. Maciel NM, Conti MHS, Simeão SFAP. Corrente JE, Ruiz T, De Vitta A. Morbidades referidas e qualidade de vida: estudo de base populacional. Fisioter Pesqui. 2016;23(1):917. https://doi.org/10.1590/1809-2950/14817923012016.

23. Amaral TLM, Amaral CA, Lima NS, Vasconcelos HP, Prado PR, Monteiro GTR. Multimorbidade, depressão e qualidade de vida em idosos atendidos pela estratégia de saúde da família em Senador Guiomard, Acre, Brasil. Ciênc. Saúde Coletiva. 2018;23(9):3077-84. https://doi. org/10.1590/1413-81232018239.22532016.

24. Vera ECBA. Terapia medicamentosa do idoso: fatores de influência [dissertação]. São Paulo: Pontífica Universidade Católica, Programa de Estudos Pós- graduados em Gerontologia; 2017. Icapturado 2020 Mar 02]. Disponivel em: https://sapientia.pucsp.br/ bitstream/handle/19829/2/Elaine\%20Cristina\%20 Biffi\%20Alonso\%20Vera.pdf.

25. Melchiors AC, Correr CJ, Pontarolo R, Santos FOS, Souza RAP. Qualidade de vida em pacientes hipertensos e validade concorrente do Minichal-Brasil. Arq Bras Cardiol. 2010; 94(3):357-64. https://doi.org/10.1590/ S0066-782X2010000300013.

26. Junior TD, Cunha CLP, Cerci RJ, Stier Jr AL, Vitola JV. Estudo de perfusão miocárdica em obesos sem doença cardiaca isquêmica conhecida. Arq Bras Cardiol. 2019; 112(2):121-28.

27. Andrade JMO, Rios LR, Teixeira LS, Vieira FS, Mendes DC, Vieira MA, Silveira MF. Influência de fatores socioeconômicos na qualidade de vida de idosos hipertensos. Ciênc. Saúde Coletiva. 2014:19(8):3497-504. https://doi. org/10.1590/1413-81232014198.19952013.

28. Almeida-Brasil CC, Silveira MR, Silva KR, Lima, Marina Guimarães, Faria, C DCM, Cardoso, CL, Menzel, H-JK, Ceccato MGB. Qualidade de vida e características associadas: aplicação do WHOQOL-BREF no contexto da atenção primária à saúde. Ciênc. Saúde Coletiva. 2017; 22(5):1705-16. https://doi.org/10.1590/141381232017225.20362015 .

29. Miranda LCV, Soares SM, Silva PAB. Qualidade de vida e fatores associados em idosos de um centro de referência à pessoa idosa. Ciênc. Saúde Coletiva. 2016;21(11): 353344. https://doi.org/10.1590/1413-812320152111.21352015.

30. Ribeiro CG, Ferretti F, Sá CA. Qualidade de vida em função do nível de atividade física em idosos urbanos e rurais. Rev Bras Geriatr Gerontol. 2017; 20(3):330-9. https://doi.org/10.1590/1981-22562017020.160110. 


\section{$8 / 8$ PAJAR, Porto Alegre, v. 8, p. 1-8, jan.-dez. $2020 \mid$ e-35631}

Henrique Souza Barros de Oliveira

Médico pelo Centro Universitário São Camilo (CUSC, São Paulo, SP, Brasil).

Leticia Silva de Jesus

Graduanda em Medicina pelo Centro Universitário São Camilo (CUSC, São Paulo, SP, Brasil).

\section{Diego Reses de Gino}

Graduando em Medicina pela Universidade Nove de Julho (UNINOVE, Guarulhos, SP, Brasil).

\section{Maria Elisa Gonzalez Manso}

Doutora em Ciências Sociais pela Pontifícia Universidade Católica de São Paulo (PUC-SP, São Paulo, SP, Brasil), professora do Centro Universitário São Camilo (CUSC-SP) em São Paulo, SP, Brasil

\section{Endereço para correspondência}

Maria Elisa Gonzalez Manso

Rua Celso de Azevedo Marques, 740, apto 1001

Parque da Mooca, 03122010

São Paulo, SP, Brasil 